\title{
Effects of Anaesthesia on Atrial Fibrillation Organization during Catheter Ablation Procedures
}

\author{
R Cervigón ${ }^{1}$, J Moreno² ${ }^{2}$ C Heneghan ${ }^{3}$, J Mateo ${ }^{1}$, \\ C Sánchez ${ }^{1}$ \\ ${ }^{1}$ Group of Bioengineering Innovation (GIBI). University of Castilla-La Mancha, Cuenca, Spain \\ ${ }^{2}$ Unidad de Arritmias. Hospital Clínico San Carlos, Madrid, Spain \\ ${ }^{3}$ University College Dublin, Dublin, Ireland
}

\begin{abstract}
Effect of anaesthetic agents in restoration rhythm procedures during atrial fibrillation (AF) has not been fully investigated. Intra-atrial recordings belong to 27 patients diagnosed with $A F$ were analyzed "before" (baseline) and "during" anaesthetic infusion. Organization measurements were evaluated in time-domain and frequencydomain, as well as approaches from non linear analysis. The frequency domain measurements showed that the average AF cycle length increased as an effect of anaesthesia. The results of time and frequency linear organization measurements showed a higher correlation during the anaesthetic infusion in the right atrium with an opposite effect in the left atrium. The same effects were corroborated with entropy analysis. The proposed methodology provide additional insights to the understanding of the role of the anaesthetic, by suggesting increased parasympathetic activation at atrial level.
\end{abstract}

\section{Introduction}

$\mathrm{AF}$ is characterized by an abnormal excitation of the atria where multiple, rapidly changing and spatially disorganized activation wavelets sweeping across the surface of atria, forming complex and ever-changing patterns of electrical activity [1,2].

Since $\mathrm{AF}$ is associated with an elevated heart rate, a possible treatment strategy is heart rate control. Although there is still clinical controversy over whether rhythm control, and therefore conversion to normal sinus rhythm is more desirable.

Electrical Cardioversion (ECV) and radiofrequency (RF) ablation of ectopic beats from pulmonary veins are at present, the methods more frequently used in patients with paroxysmal AF to restore normal sinus rhythm [3].
During these procedures, along with cardiac electrophysiological studies, the patients are under the influence of anaesthetic agents. One of the anaesthetics more often used is propofol. Propofol (2,6-diisopropylphenol) is a new, rapidly acting intravenous anaesthetic. The rapid redistribution and metabolism of propofol, resulting in a short elimination half-life of approximately one hour, suggest that the drug could be suitable for use during short surgical procedures. There is now the recent interest whether, and if so how, propofol affects the electrophysiological properties of the atria.

The purpose of this study was, therefore, to explore the influence of anaesthesia on the electrical activity organization within the atria in patients with $\mathrm{AF}$, through the characterization of the intra-atrial recordings (IEGs) in time-domain and frequency-domain with linear and with nonlinear dynamics approaches.

\section{Materials}

History of AF before the ablation procedure was recorded in 27 patients (21 paroxysmal $\mathrm{AF}$ and 6 persistent $\mathrm{AF}$ ) before and under anesthesia with propofol (bolus of $1.5-2 \mathrm{mg} / \mathrm{kg}$ intravenously with incremental doses depending on the weight and time to hypnosis).

A bipolar catheter was positioned in the high right atrium, and a 24-pole catheter (Orbiter, Bard Electrophysiology, 2-9-2 mm electrode spacing) was positioned in the right atrium (RA) with the distal dipoles into the coronary sinus (CS) to record left atrium (LA) electrical activity as well. Since these are bi-polar electrograms, this results in a set of 12 signals, which we refer to as dipole $1-2$, dipole 3-4, etc. While the exact positioning of the catheter will vary from subject to subject, in general the leads 1-2, 3-4, and 5-6 are in the LA, leads 7-8, 9-10, 11-12 are in the septum area (SA), and leads from 15-16, 17-18, ... to 23-24 are in the RA. 


\section{Methods}

In order to characterize these signals, at first a preprocessing stage was applied, followed to the extraction of different organization indexes from time domain, as interelectrode cross-correlation and entropy measurement and from frequency domain, as coherence spectrum and irregularity index extracted from the spectrum.

\subsection{Signal preprocessing}

IEGs recordings signals, without any influence of ventricular activation, were band-pass filtered using a 40 to $250 \mathrm{~Hz}$ third-order Butterworth filter. The resulting filtered waveforms were then rectified, and filtered once more using a 20-Hz low-pass third-order Butterworth filter. This preprocessing extracts a time varying waveform proportional to the amplitude of the high-frequency components in the original atrial electrogram, enhancing the periodicity or nonperiodicity of the signals. This algorithm was used to take a complex waveform and transform it to a series of atrial activations while diminishing the effects of changing electrogram morphology and/or amplitude $[4,5]$.

\subsection{Spectral analyses}

Spectral analysis was performed before and after propofol infusion. Power spectra was quantified by measuring the peak frequency signal, it was converted to a cycle length (FCL 1.000/frequency (ms)). This was averaged from the epochs resulted from the length sequences divided in 4096 points with a overlap of $50 \%$.

An area ratio of the spectral area over a $1 \mathrm{~Hz}$ window around the maximum power to the total spectral area from 3 to $9 \mathrm{~Hz}$ was termed the fibrillation regularity index (RI), that exhibits the ratio of the power at the larger peak and adjacent frequencies outside this frequency band.

\subsection{Cross-correlation analysis}

Botteron and Smith tested the hypothesis that atrial activations sequences within some small region are wellcorrelated (reflective of their participation is the same wavelet), while activation sequences beyond some distance are no longer well-correlated (reflective of their participation in different wavelets). They found that the atrial activation processes during AF are spatially correlated with the degree of correlation, decaying monotonically with the distance of the bipoles, as these sequences did not belong to the same wavefront (eq. 1) $[4,6]$.

$$
R_{k}=\frac{\frac{1}{n} \sum_{i=k+1}^{n}\left(x_{i}-\bar{x}\right)\left(x_{i-k}-\bar{x}\right)}{\frac{1}{n} \sum_{i=k+1}^{n}\left(x_{i}-\bar{x}\right)^{2}}
$$

The cross-correlation function was calculated over a range of lag intervals for each electrogram combination (non-overlapping segments of $1-1.5 \mathrm{~s}$.). For each data segment analyzed, the absolute peak was considered the correlation coefficient representing the degree of correlation between those two signals for that period of time. This operation was repeated on sequential data segments for the entire data file allowing the construction of a correlation average for the different activation times of the signal across electrograms.

\subsection{Coherence spectrum}

Spectral analysis of IEGs during AF was first motivated by the need of automated arrhythmia discrimination schemes [7], since it may reflect properties and structures which can not be easily detected in the time-domain.

$\mathrm{AF}$ is characterized by disorganized, continually changing patterns of activation and the absence of a constant temporal relationship between multiple sites on the heart. This continually changing temporal or phase relationship may be quantified in the frequency domain by magnitude squared coherence (Eq. 2) [8]. This method quantifies temporal and spatial organization during $\mathrm{AF}[8,9,10,11]$, measuring of the constancy of the time delay (phase) at a specific frequency between two endocardial electrograms ( $\mathrm{x}$ and $\mathrm{y}$ ) as a function of frequency (eq. 2).

$$
M S C(f)=\left|S_{x y}(f)\right|^{2} / S_{x x}(f) S_{y y}(f)
$$

The data were partitioned into 4096 points segments (with a $50 \%$ overlap). Each window was then multiplied by a Hanning window and Fourier transformed. Coherence was defined as the magnitude-squared of the cross spectrum between the two signals divided by the auto-power spectra of the signals from $0-20 \mathrm{~Hz}$.

\subsection{Sample entropy}

The activation patterns behind the electrical activity of the heart during AF have often been characterized as random phenomena. Entropy measurements have been recently used to the analysis understanding of complex physiological time series, particularly to quantify the regularity of the wavefront in cardiac tissues.

In this study, we have used Sample Entropy (SampEn) [12] as a characterization of activation patterns in a determined atrial area. It can be calculated by the equation 3 , where $\mathrm{B}$ is a total number matched $\mathrm{n}$ patterns, and $\mathrm{A}$ is the total number of matched $m+1$ patterns.

$$
\text { SampEn }=-\ln \frac{B}{A}
$$

SampEn is equal to the negative natural logarithm of an estimate of the conditional probability that sub-series 
(epochs) of length $m$ that match point-wise within a tolerance $r$ also match at the next point. Larger SampEn values indicate greater independence, less predictability, hence greater complexity in the data. This, in turn, may imply that decreased complexity or greater regularity in the time series is not associated with the disease.

For the study discussed in this paper, SampEn is estimated using the widely established parameter values of $m=2$, and $r=0.25 \sigma$, where $\sigma$ represents the standard deviation of the original data sequence, as suggested by Pincus [13].

\section{Results}

\subsection{Spectral analyses results}

Propofol was found to increase atrial refractoriness, thereby providing a mechanism to increase the wavelength during AF. The values of FCL were found to be larger during propofol infusion with respect to basal conditions $(171.98 \pm 13.43 \mathrm{~Hz}$ vs. $174.49 \pm 15.26 \mathrm{~Hz}$, respectively $p=0.121)$.

AF regularity using the RI parameter showed a slightly higher organization during propofol infusion than at baseline state $(0.589 \pm 0.05$ vs. $0.569 \pm 0.05 ; p=0.062$, respectively).

\subsection{Cross-correlation results}

As pointed out in Section 3.3, the estimate of the correlation for the same inter-electrode separation was averaged, resulting in a single correlation versus distance relationship.

In order to have electric activations at the same instant, before correlation calculation, the original leads were aligned by the maximum amplitude in a period time.

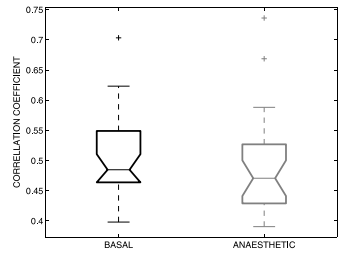

(a)LA Correlation

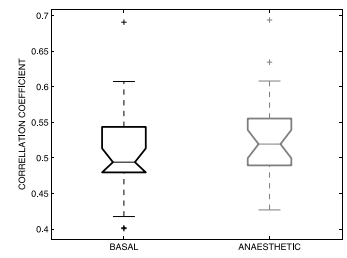

(b)RA Correlation
Figure 1. The correlation coefficient at each interbipole separation with the correlation defined as one at zero distance across all subjects (basal (black), propofol (grey))

The correlation coefficient was calculated at the closest inter-electrode distance, to evaluate the difference in correlation along the atrial area. From this analysis it is possible observe that during the anaesthetic infusion the correlation is greater in the RA, with the opposite effect observed in the LA, with $0.51 \pm 0.07$ at basal vs. $0.49 \pm 0.08$ at propofol peak and in RA $0.51 \pm 0.06$ vs. $0.53 \pm 0.06$ respectively $(p=0.008)$ (Figure 1).

\subsection{Coherence spectrum results}

Coherence sensitivity at the closest inter-electrode distance was unable to distinguish between the two states studied. In LA $0.28 \pm 0.11$ at basal vs. $0.26 \pm 0.09$ at propofol state and in RA $0.23 \pm 0.07$ vs. $0.25 \pm 0.07$ respectively, $p=0.008$ (Figure 2 ).

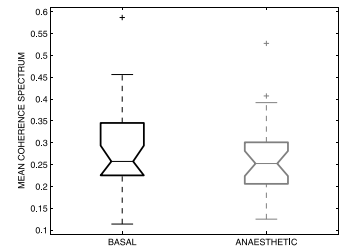

(a)LA Coherence

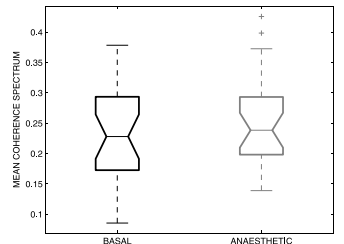

(b)RA Coherence
Figure 2. Mean coherence spectrum in LA and RA across all subjects, (basal (black), propofol (grey))

\subsection{Entropy results}

The SampEn of the time series from the intra-atrial recordings was found to be significantly reduced in the RA during propofol infusion compared with the basal state $(0.066 \pm 0.014$ at basal vs. $0.063 \pm 0.014$, respectively $)$ and the opposite effect was found in the period in the LA, $0.078 \pm 0.016$ at basal vs. $0.081 \pm 0.016$, at propofol peak, $(p=0.023)$ (Figure 3).

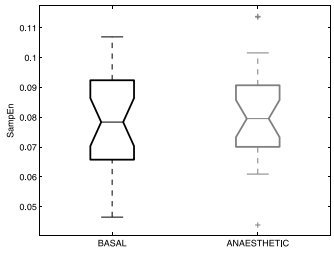

(a)LA SampEn

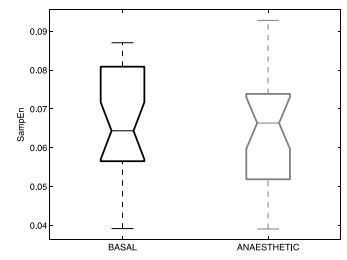

(b)RA SampEn
Figure 3. SampEn of dipole electrode recording time series $\mathrm{AF}$ in $\mathrm{LA}$ and $\mathrm{RA}$ across all subjects, (basal (black), propofol (grey)) 


\section{Discussion and conclusions}

The influence of propofol on the electrophysiological properties of the myocardium is sparsely reported in the literature [14, 15]. Quantification effects of anaesthetics, such as propofol, can be helpful to explain the behavior of nervous system in AF pathology.

The changes in the spectral content of IEGs showed a larger cycle length and regularity index during propofol infusion, and could suggest a decrease in atrial refractoriness during the administration of anaesthetic agent.

Between the methods used to quantify the spatiotemporal organization during $\mathrm{AF}$, the analysis of time series, in particular showed that correlation inter-electrodes had an increased organization in the RA during propofol administration. Furthermore, the coherence spectrum was used to asses the phase relation between two simultaneous electrogram recordings at multiple atrial sites, showing a similar trend. Both methods showed a fairly organized wavefront in RA compared with a less organized or periodic activity in the LA.

The entropy measurement put into evidence that RA had a higher regularity at propofol peak than at basal state with a opposite effect in the LA. These results may be extrapolated to paroxysmal and persistent $\mathrm{AF}$, with a more homogeneous distribution in persistent $\mathrm{AF}$ and larger differences in LA in patients with paroxysmal AF.

Our findings suggest that propofol induces an increase in atrial spatial organization and regularity in RA, exerting a parasympathetic activation at regional atrial level.

More information about the effect of individual intraoperative factors may be helpful to evaluate these phenomenas, and also to have a better understanding of the behavior of ANS in AF, verifying if the differences between electrophysiological properties before and during anaesthetic infusion are due to true changes in physiological conditions.

\section{Acknowledgements}

This work was supported by Castilla-La Mancha research scheme (Ref: PAC-05-008-1) and from the Ministery of Education and Science of Spain (Ref: TEC200508401-C02-01).

\section{References}

[1] M Allessie KK, Wijffels M. Atrial arrhythmias: State of the art. DiMarco, J.P. and Prytowsky, E.N. Eds., Futura Publishing Company, Armonk, NY, 1995; 155-161.

[2] Bollmann A, Sonne K, Esperer H, Toepffer I, Langberg J, Klein H. Non-invasive assessment of fibrillatory activity in patients with paroxysmal and persistent atrial fibrillation using the holter ECG. Cardiovascular Research 1999;44:6066.
[3] Haissaguerre M, Jais P, Shah D, Takahashi A, Hocini M, Quiniou G, Garrigue S, Mouroux AL, Metayer PL, Clementy J. Spontaneous initiation of atrial fibrillation by ectopic beats originating in the pulmonary veins. N Engl J Med 1998;339(10):659-666.

[4] Botteron G, Smith J. A technique for measurements of the extent of spatial organization of atrial activation during atrial fibrillation in the intact human heart. IEEE Trans Biom Eng 1995;42:579-586.

[5] Thomas H, Everett I, Kok LC, Richard H, Vaughn J, Randall M, Haines E. Quantitative assessment of the spatial organization of atrial fibrillation in the intact human heart. Circulation 1996;93:513-518.

[6] Botteron G, Smith J. Quantitative assessment of the spatial organization of atrial fibrillation in the intact human heart. Circulation 1996;93:513-518.

[7] Slocum J, Sahakian A, Swiryn S. Computer discrimination of atrial fibrillation and regular atrial rhythms from intra-atrial electrograms. Pacing Clin Electrophysiol 1988; 11:610-621.

[8] Ropella K, Sahakian A, Baerman J, Swiryn S. The coherence spectrum: A quantitative discriminator of fibrillatory and nonfibrillatory cardiac rhythms. Circulation 1989; 80:112-119.

[9] Ropella K, Baerman J, Sahakian A, Swiryn S. Differentiation of ventricular tachyarrhythmias. Circulation 1990; 82:20352043.

[10] Lovett E, Ropella K. Time-frequency coherence analysis of atrial fibrillation termination during procainamide administration. Ann Biomed Eng 1997;25:975-984.

[11] Sih H, Ropella K, Swiryn S, Gerstenfeld E, Sahakian A. Observations from intraatrial recordings on the termination of electrically induced atrial fibrillation in humans. Pacing Clin Electrophysiol 1994;17:1231-1242.

[12] Richman J, Moorman J. Physiological time-series analysis using approximate entropy and sample entropy. Am J Physiol Heart Circ Physiol 2000;278:2039-2049.

[13] Pincus S, Acad A. Approximate entropy (apen) as complexity measure. Sci 2001;954:245.

[14] Briggs I, Heapy C, Pickering L. Electrophysiological effects of propofol on isolated sinoatrial node preparations and isolated atrial conduction in vitro. $\mathrm{Br} \mathbf{J}$ Pharmacol 1989;97:504-P.

[15] Zaballos M, Almendral J, Anadn M, Gonzlez M, Navia J. Comparative effects of thiopental and propofol on atrial vulnerability: electrophysiological study in a porcine model including acute alcoholic intoxication. British Journal of Anaesthesia 2004;93:414-421.

Address for correspondence:

Raquel Cervigón Abad

E.U.P. Camino del Pozuelo sn

Campus Universitario

16071 Cuenca 